\title{
Propiedades psicométricas de una versión española breve de 30 ítems del Cuestionario de Ansiedad y Depresión (MASQE30**
}

\section{Psychometric Properties of the Spanish Version of 30-Item Brief Depression and Anxiety Symptoms Questionnaire (MASQS30)}

Recepción: 17 Septiembre 2015 | Aprobación: 05 Mayo 2017

\author{
Manuel GonzÁlez \\ Universidad de la Laguna, España \\ ORCID: http://orcid.org/0000-0001-9083-4233 \\ IGNACIO IBÁÑEZ \\ Universidad de la Laguna, España
}

a Autor de correspondencia. Correo electrónico: mgonzaro@ull.es

Para citar este artículo: González, M., \& Ibáñez, I. (2018). Propiedades psicométricas de una versión española breve de 30 ítems del Cuestionario de Ansiedad y Depresión (MASQE30). Universitas Psychologica, 17(1), 1-10. https://doi.org/10.11144/Ja veriana.upsy17-1.ppve

\section{RESUMEN}

Se presenta en esta investigación una versión corta de 30 ítems del Mood and Anxiety Symptoms Questionnaire ([MASQ]; Cuestionario de Síntomas de Ansiedad y del Humor) diseñado para medir las tres dimensiones del modelo tripartito de la ansiedad y la depresión, a saber, el Afecto Negativo (AN), el Afecto Positivo (AP) y la Ansiedad Somática (AS). Esta adaptación es una versión holandesa que se administró a una muestra de la población general canaria. Los objetivos propuestos son conocer la validez de constructo del MASQE30, mediante análisis factoriales exploratorio y confirmatorio, así como sus propiedades psicométricas y la validez convergente, discriminante y predictiva con ansiedad y depresión evaluadas con cuestionarios. Los resultados confirman la adecuada validez de constructo del MASQE30, así como coeficientes de consistencia interna que oscilan entre 0.88 y 0.92 y una estabilidad temporal que va desde 0.47 a 0.68 . Los resultados corroboran que el AN y AS son compartidos por la ansiedad y la depresión, y el AP es específico de la depresión. El AN y AP corroboran las predicciones del modelo, pero no el resultado de AS. Se propone el MASQE30 como un instrumento adecuado para el estudio dimensional de la depresión y la ansiedad en población clínica y comunitaria.

Palabras clave

modelo tripartito; afecto negativo; afecto positivo; ansiedad somática.

\begin{abstract}
In this investigation, we present a short 30-item version of the Mood and Anxiety Symptoms Questionnaire (MASQS30), designed to measure the three dimensions of the tripartite model of anxiety and depression, namely, negative affect (NA), positive affect (PA), and somatic anxiety (SA). This adaptation is from a Dutch version that we administered to a sample of the general canarian population. The proposed goals are to determine the construct validity of the MASQS30 through exploratory and confirmatory factor analysis, as well as its psychometric properties, and convergent, discriminant, and predictive validity with anxiety and depression as assessed through questionnaires. The results confirm adequate construct validity of the MASQS30, as well as internal
\end{abstract}


consistency coefficients ranging between 0.88 and 0.92 , and temporal stability, ranging from 0.47 to 0.68 . The results corroborate that NA and SA are shared by anxiety and depression, whereas PA is specific to depression. NA and PA corroborate the predictions of the model, but the result of SA does not. The MASQS30 is proposed as an adequate instrument for the dimensional study of depression and anxiety in clinical and community population.

Keywords

tripartite model; negative affect; positive affect; physiological hyperarousal.

Los trastornos de ansiedad y del humor poseen la prevalencia más elevada de los problemas de salud mental en atención primaria (Kessler et al., 2005). Se estiman en el $20 \%$ de la población mundial, causando una incapacidad sustancial y se convertirán en la principal causa de carga de enfermedad para el año 2020 en los países desarrollados (Organización Mundial de la Salud [OMS], 2001).

En la adquisición y el mantenimiento de los trastornos emocionales podemos encontrar dos tipos de vulnerabilidades. Una vulnerabilidad temperamental, que señala a algunos rasgos de la personalidad, como el neuroticismo/ afectividad negativa y la extraversión/afectividad positiva, y las vulnerabilidades cognitivas, que son características parecidas a los rasgos estables de personalidad que interactúan con el estrés significativo de la vida, confiriéndole responsabilidad en los trastornos emocionales (Reardon \& Williams, 2007). La posibilidad de que diversos trastornos compartan elementos de vulnerabilidad enlaza directamente con el concepto de proceso transdiagnóstico, que hace referencia a la existencia de un factor de riesgo común o a un proceso que pudiera estar en la etiología y el mantenimiento de diversos trastornos emocionales (Egan, Wade, \& Shafran, 2011).

El interés con respecto a la naturaleza de la depresión y la ansiedad ha recibido una amplia atención en la bibliografía científica (Anderson \& Hope, 2008), y para la explicación de la co-ocurrencia entre ambos constructos se ha propuesto el modelo tripartito (Clark \& Watson, 1991a). Las tasas de comorbilidad entre los trastornos emocionales tienen serias implicaciones para el diagnóstico, la priorización y diseminación de los tratamientos (EhrenreichMay \& Bilek, 2012).

Respecto a su manifestación, la ansiedad y la depresión son aparentemente distintas, pues la ansiedad se centra en la emoción del miedo; en contraste, la depresión parece dominada por la emoción de tristeza (Buckby, Cotton, Cosgrave, Killackey, \& Yung, 2008). Esta supuesta distintividad se enfrenta con las dificultades que se han encontrado para diferenciar de forma empírica ambos constructos. La validez en la distinción conceptual entre la ansiedad y la depresión invariablemente se ha cuestionado, pues los estados de ánimo depresivo y ansioso a menudo co-ocurren y sus síntomas claves muestran un solapamiento sustancial que implican unos factores clínicos y etiológicos comunes (Mineka, Watson, \& Clark, 1998).

En la actualidad existen varias medidas de autoinforme para evaluar la ansiedad y la depresión. Sin embargo, los datos empíricos han observado algunas limitaciones de esos instrumentos, estableciendo la necesidad de nuevas y mejores medidas para evaluarlos. Los instrumentos que evalúan ansiedad y depresión están altamente correlacionados, dando lugar a una validez discriminante modesta (Clark \& Watson, 1991a). En este sentido: 1) las escalas basadas en listas de adjetivos no proporcionan medidas discriminantes del estado de ánimo depresivo y ansioso (Clark \& Watson, 1991a); 2) las escalas correlacionan entre 0.62 y 0.7 a través de un amplio rango de muestras de pacientes y no clínicas, mostrando un elevado solapamiento en contenidos (Chorpita \& Daleiden, 2002) y 3) aproximadamente, la mitad de los pacientes diagnosticados de un trastorno depresivo o de ansiedad manifiestan síndromes relativamente puros de uno u otro tipo de trastorno.

Para conocer las relaciones entre ansiedad y depresión se ha propuesto el Modelo Tripartito de la Ansiedad y Depresión (Clark \& Watson, 1991a), que explica tanto las características distintivas como las solapadas entre los dos constructos. Este modelo parte de la Teoría 
del Afecto (Tellegen, 1985), que considera a este último constituido por dos dimensiones básicas, el afecto positivo (AP) y el afecto negativo (AN), y al cual Clark y Watson (1991a) añadieron una tercera dimensión, que parece ser relativamente específica de los trastornos de ansiedad, la ansiedad somática (AS). Esta última se caracteriza por una serie de manifestaciones de tensión y activación somática. El AN se asocia a múltiples variables, entre las que se encuentran el nerviosismo, el pesimismo y la baja autoestima (González, Herrero, Viña, Ibáñez, \& Peñate, 2004). El AP refleja la medida en que una persona se siente activa, entusiasta; por el contrario, el bajo AP o anhedonia se caracteriza por fatiga, falta de interés por las cosas (Clark \& Watson, 1991a). Así, el nerviosismo, la tensión y la preocupación parece estar más relacionada con la ansiedad, por el contrario, la cólera, la culpabilidad y la tristeza lo hacen con depresión (Buckby et al., 2008).

El modelo tripartito señala que los trastornos depresivos y de ansiedad comparten un factor general de un elevado AN. Por otra parte, ambos trastornos poseerían también una serie de síntomas, así la ansiedad somática (AS) es especifica de los trastornos de ansiedad y la anhedonia o bajo AP lo es de la depresión (Clark \& Watson, 1991a). Para evaluar empíricamente su modelo, los autores desarrollaron un autoinforme de 90 ítems, el Cuestionario de Síntomas de Ansiedad y del Humor (Watson et al., 1995).

Las investigaciones sobre la validez de constructo del MASQ90 con muestras clínicas y población normal, donde se ha empleado el análisis factorial exploratorio, han encontrado una adecuada validez de constructo de las tres escalas (de Beurs, den Hollander-Gijsman, Helmich, \& Zitman, 2007). Sin embargo, cuando se ha empleado el análisis factorial confirmatorio, los datos no fueron adecuados para las tres dimensiones, encontrando una estructura bidimensional (Boschen \& Oei, 2007; Buckby et al., 2008).

Estos resultados del análisis factorial exploratorio formulan algunas deficiencias en el MASQ90. Así, 1) un tercio de los ítems mostraban saturaciones débiles o complejas (de
Beurs et al., 2007), de manera que la eliminación de estos ítems podrían mejorar la validez del MASQ90 (Boschen \& Oei, 2007); 2) de los 90 ítems del cuestionario se incluyen 77, sugiriendo que no todos son necesarios para la formulación del modelo; 3) los resultados del análisis factorial exploratorio mostraron saturaciones que difieren de su predicción teórica; 4) varios ítems fueron colocados incorrectamente y 5) el factor de AP fue definido muy claramente por ítems positivos del afecto. Otras dificultades se refieren a su validez discriminante moderada, dado la alta validez convergente entre ansiedad y depresión, esta co-ocurrencia se ha observado en muestras de la población general y clínicas, no solo en medidas de autoinforme, sino también en escalas de calificación de médicos, padres y maestros (de Beurs et al., 2007; Mineka et al., 1998).

Estos resultados sugieren de alguna manera una propuesta de mejoras y modificaciones en el MASQ90. Así, en un intento de paliar las limitaciones anteriores, se han elaborado versiones cortas, como el MASQ62 que está constituido por 62 ítems (Clark \& Watson, 1991b) y el Mini-Masq26 que consta de 26 ítems (Casillas \& Clark, 2000).

Recientemente se ha publicado una versión holandesa de 30 ítems, el MASQD30 (Wardenaar et al., 2010), cuyas propiedades psicométricas fueron investigadas y comparadas entre los datos obtenidos y el MASQ90, alcanzando un modelo de tres dimensiones. Las escalas del MASQD30 demostraron adecuadas propiedades psicométricas (tanto en muestras clínicas como de la población general), siendo los coeficientes de consistencia interna de 0.91 para AN, 0.93 para AP y 0.87 para AS. En cuanto a la validez convergente y discriminante, el AN correlacionó 0.78 con el BDI-II y 0.6 con el BAI. La AS muestra coeficientes de correlación con el BAI de 0.76 y 0.55 con el BDI-II. En cuanto al AP o Depresión Anhedonia, correlaciona 0.56 con el BDI-II y 0.49 con el BAI. Estos coeficientes son muy similares a los del MASQ90, señalando que la validez convergente y discriminante del MASQ90 se preserva en el MASQD30 (Wardenaar et al., 2010). 
Así las cosas, los objetivos de esta investigación son conocer la validez de constructo, así como las propiedades psicométricas de una versión corta de 30 ítems (el MASQE30) en una muestra de población general de Canarias.

\section{Método}

\section{Participantes}

El número total de personas que participaron en esta investigación fue de 493, residentes en la isla de Tenerife (Islas Canarias), donde el 61.3 \% eran mujeres y el $38.7 \%$ hombres. El rango de edad osciló entre los 18 y 73 años, con una media de 29.08 años $(D E=12.13)$ y una moda de 23. Con respecto al estado civil, el $66.2 \%$ eran solteros, un $24.7 \%$ casados, el $6.8 \%$ divorciados/separados y un $2.3 \%$ eran parejas de hecho. Si se tiene en cuenta el nivel de estudios, un $13.6 \%$ poseían estudios de educación general básica (EGB), un $32.5 \%$ estudios de BUP (bachillerato unificado y polivalente) y COU (cuso de orientación universitaria), un $12 \%$ de FP (formación profesional), el $29.6 \%$ poseían estudios medios y un $12.2 \%$ estudios superiores. Pasados cinco semanas, un total de 80 personas volvieron a cumplimentar el MASQE30, con la finalidad de obtener la fiabilidad test-retest del cuestionario.

\section{Instrumentos}

- Inventario de Ansiedad de Beck (Beck Anxiety Inventory [BAI]; Beck, Epstein, Brown, \& Steer, 1988): Es un inventario de 21 ítems que evalúa el nivel de gravedad de la agorafobia y pánico. $\mathrm{Su}$ fiabilidad test-retest es de 0.75 y la consistencia interna de 0.92 .

- Inventario de Depresión de Beck-II (Beck Depression Inventory-II [BDI-II]; Beck, Steer, \& Brown; 1996) traducido al español por Sanz, Perdigón y Vásquez (2003): La consistencia interna es de 0.89 .

- Cuestionario de Sintomas de Ansiedad
y Depresión (Mood and Anxiety

Symptom Questionnaire [MASQ], Watson et al.,
1995): En este caso, se empleó una adaptación holandesa de 30 ítems, el MASQD30 (Wardenaar et al., 2010). Está constituido por 30 ítems extraídos de los criterios para los trastornos de ansiedad y del estado de ánimo, que se agrupan en tres escalas, siguiendo el modelo tripartito. Los coeficientes de consistencia interna son $\alpha=0.91$ para $\mathrm{AN} ; \alpha=$ 0.93 para AP y $\alpha=0.87$ para AS.

\section{Procedimiento}

Formando parte del programa de prácticas voluntarias de Técnicas de Exploración Psicológica, 40 alumnos fueron entrenados en la administración en las distintas pruebas. Además, a los alumnos se les informó que seleccionaran de su entorno cercano a un grupo de ocho personas, mayores de 18 años balanceados por género, lo que se denomina técnica de muestreo de bola de nieve. Una vez contactadas esas personas, se les informaba del carácter experimental del trabajo y se les pedía su participación voluntaria, previa información y firma del consentimiento informado.

Análisis estadístico de los datos

Para conocer la validez de constructo del MASQE30, se recurrió tanto al análisis factorial exploratorio como el confirmatorio.

\section{Resultados}

Se presentan a continuación los resultados de la validez de constructo, convergente y discriminante, así como las propiedades psicométricas del MASQ30. Pero es necesario matizar una pequeña modificación que se realizó en el MASQE30, tal como sustituir el ítem tres "Me sobresalté fácilmente", por el ítem 69 "Mis músculos se crisparon o temblaron". 
Validez estructural y de constructo

\section{Análisis Factorial Exploratorio}

Se someten los 30 ítems a un análisis factorial exploratorio de componentes principales y rotación Promax, empleando el método de estimación WLSMV del Mplus versión 3.0 (Muthen \& Muthen, 1998). No obstante, frente a este criterio se valoraron los resultados de dos procedimientos más fiables para resolver la cuestión de cuántos factores retener y rotar (Velicer, Eaton, \& Fava, 2000): el Test de mínima correlación parcial promediada (Minimum Average Partial [MAP]; Velicer, 1976) y el Análisis paralelo (Parallel Analysis [PA]; Horn, 1965). En la Tabla 1, atendiendo a los criterios anteriores, se retuvieron tres factores que explican el 39.7 \% de la varianza. El Factor 1 se denominó AN (afecto negativo), el Factor 2, AS (Ansiedad Somática) y el Factor 3, AP (Afecto Positivo). Estos resultados se pueden observar en la Tabla 1.
Tabla 1

Análisis factorial exploratorio para el MASQE30 (método de estimación WLSMV) $N=493$

\begin{tabular}{|c|c|c|c|c|c|c|c|}
\hline \multicolumn{8}{|c|}{ Estimación WLSMV } \\
\hline Ítem & AN & AS & AP & $r_{x v}$ & $\alpha$ & $M$ & $D E$ \\
\hline 23. Tuve problemas para tomar decisiones & 0.68 & & & 0.75 & 0.81 & 1.9 & 1.2 \\
\hline 21. Me sentía inferior a otros & 0.73 & & & 0.77 & 0.81 & 1.6 & 1 \\
\hline 28. Me preocupé mucho por las cosas & 0.5 & & & 0.44 & 0.82 & 2.6 & 1.2 \\
\hline 01. Me sentía confundido & 0.7 & & & 0.67 & 0.81 & 2.1 & 1.1 \\
\hline 08. Me sentía desesperanzado & 0.58 & & & 0.46 & 0.81 & 1.6 & 1.1 \\
\hline 11. Me sentia insatisfecho con todo & 0.57 & & & 0.57 & 0.81 & 1.7 & 1 \\
\hline 04. Me sentía inútil & 0.66 & & & 0.68 & 0.81 & 1.5 & 1.1 \\
\hline 06. Me sentía irritable & 0.64 & & & 0.62 & 0.81 & 1.9 & 1 \\
\hline 10. Me culpé por muchas cosas & 0.66 & & & 0.56 & 0.81 & 1.7 & 1.1 \\
\hline 14. Me sentia pesimista acerca del futuro & 0.56 & & & 0.66 & 0.81 & 1.9 & 1.2 \\
\hline 18. Me sentía con vértigos o mareos & & 0.68 & & 0.31 & 0.81 & 2.5 & 1.1 \\
\hline 02. Sentía náuseas & & 0.54 & & 0.53 & 0.82 & 1.3 & 0.79 \\
\hline 15. Tuve un dolor en el pech & & 0.84 & & 0.36 & 0.81 & 2.1 & 1.1 \\
\hline 30. Tuve problemas para tragar & & 0.71 & & 0.5 & 0.81 & 1.3 & 0.79 \\
\hline 22. Mis músculos se cr & & 0.83 & & 0.52 & 0.81 & 1.4 & 0.92 \\
\hline 19. Tuve la respiración entrecortada & & 0.78 & & 0.47 & 0.81 & 1.5 & 1 \\
\hline 26. Tuve estremecimientos o temblores & & 0.72 & & 0.48 & 0.81 & 1.5 & 1 \\
\hline 17. Tuve períodos de frío o de calor & & 0.66 & & 0.55 & 0.81 & 1.8 & 1.1 \\
\hline 27. $\mathrm{M}$ & & 0.7 & & 0.5 & 0.81 & 17 & 1.1 \\
\hline 25. Mi corazón latió aceleradamente o palpitó & & 0.61 & & 0.27 & 0.81 & 1.9 & 1.2 \\
\hline 03. Me sentía triunfador & & & 0.73 & 0.69 & 0.83 & 2 & 1.1 \\
\hline 12. $\mathrm{Se}$ & & & 0.71 & 0.66 & 0.83 & 2.4 & 1.2 \\
\hline 24. Sentía q & & & 0.67 & 0.62 & 0.83 & 2.5 & 1.1 \\
\hline 20. Me sentía realmente en plena forma, vivo & & & 0.67 & 0.72 & 0.84 & 2.6 & 1.1 \\
\hline n conmigo mismo & & & 0.66 & 0.75 & 0.83 & 2.8 & 1.1 \\
\hline 07. Me se & & & 0.67 & 0.73 & 0.84 & 2.8 & 1.1 \\
\hline 05 . Me ser & & & 0.74 & 0.69 & 0.84 & 3 & 1.1 \\
\hline 09. Me di & & & 0.69 & 0.7 & 0.84 & 2.6 & 1.2 \\
\hline 16. Me sentía muy hablador & & & 0.54 & 0.75 & 0.84 & 2.5 & 1.2 \\
\hline $\begin{array}{l}\text { 13. Sentía que tenía muchas cosas por las que } \\
\text { ilusionarme }\end{array}$ & & & 0.55 & 0.55 & 0.84 & 3.1 & 1.2 \\
\hline Medias & & & & 0.58 & 0.82 & 2.12 & 1.08 \\
\hline & 10.91 & 4.07 & 1.75 & & & & \\
\hline is factor & 0.7 & 0.68 & 0.6 & & & & \\
\hline$\% \mathrm{Va}$ & 25.4 & 10.6 & 3.7 & & & & \\
\hline$\%$ Varianza Acumulada & 25.4 & 36 & 39.7 & & & & \\
\hline
\end{tabular}

Nota. AN = Afecto negativo; AS = Ansiedad

Somática; AP = Afecto positivo; V. P. = Valor

Propio; \%V. T. = Porcentaje de varianza total, \%V.

A. $=$ Porcentaje de varianza acumulada; $r$ xy $=$ correlación ítem-total; $\alpha=$ alfa de Cronbach sin

el ítem; $M=$ Media; $D E=$ desviación estándar.

\section{Análisis Factorial Confirmatorio}

Dos cuestiones se pretenden resolver con estos análisis: la bondad de ajuste del modelo de tres factores propuesto y si es mejor incluir el ítem 3 "Me sobresalté fácilmente" o el 69 "Mis músculos se crisparon o temblaron" de la escala original en el segundo factor (AS).

En la Tabla 2, se observa que ningún ítem se distribuía de forma normal, por lo que se descartó la normalidad multivariable, empleándose procedimientos robustos a la falta de normalidad: ML robusto como método de estimación y chi-cuadrado robusta de SatorraBentler (Satorra \& Bentler, 1994). Dado que los ítems se recogen en una escala tipo Likert de 5 alternativas de respuesta y que se trataría de una variable ordinal, se emplea igualmente el método de estimación de mínimos cuadrados ponderados robustos (Weighted Least Squares Mean and Variance Adjusted [WLSMV]), adecuado para 
este tipo de datos. Los análisis se efectuaron con el Mplus 3 (Muthen \& Muthen, 1998).

La magnitud de los índices de ajuste fue evaluada siguiendo las recomendaciones de $\mathrm{Hu}$ y Bentler (1999) p > 0.95 para el Índice de Ajuste Comparativo (CFI) y el Índice de Tucker Lewis (TLI) $p<0.06$ para el error de aproximación a la raíz cuadrada media (RMSEA), $p<0.08$ para los residuales estandarizados (SRMR). En todo caso, hay que hacer notar que los puntos de corte para los índices de ajuste a un modelo no están establecidos de forma inequívoca. Así, algunos autores consideran que 0.95 es un punto de corte demasiado restrictivo cuando se emplean modelos complejos y datos reales, no simulados (Marsh, Hau, \& Wen, 2004). Para todos los modelos y métodos de estimación, todos los parámetros son significativos con $p<0.01$.

En cuanto a si es preferible optar por el ítem $3 \mathrm{o}$ el 69 del MAQ90, los datos de la Tabla 2 parecen indicar que es indiferente. Cuando se analizan las variaciones en ajuste para la escala AS, parece más adecuado retener el ítem 3 (mejores índices de ajuste o similares), pero cuando se contemplan los tres factores conjuntamente, los mejores índices se obtienen para con el ítem 69.

\section{Tabla 2}

Índices de ajuste para el MASQE30 (con ítem 690 item 3, según el caso)

\begin{tabular}{|c|c|c|c|c|c|c|}
\hline \multicolumn{2}{|c|}{ Modelo / Método Estimación } & Chi $(g l)$ & CFI & TLI & RMSEA & SRMR \\
\hline \multicolumn{7}{|c|}{ Factor a factor (cada factor por separado) } \\
\hline \multicolumn{2}{|l|}{ AN/WLSMV } & $84(27)$ & 0.955 & 0.977 & 0.069 & 0.053 \\
\hline \multicolumn{2}{|l|}{$\mathrm{AN} / \mathrm{ML}$ robusto } & $102(35)$ & 0.912 & 0.887 & 0.066 & 0.048 \\
\hline \multirow[t]{2}{*}{ AS / WLSMV } & con item 69 & $49(23)$ & 0.981 & 0.992 & 0.051 & 0.041 \\
\hline & & $43(24)$ & 0.984 & 0.992 & 0.043 & 0.043 \\
\hline \multirow[t]{2}{*}{ AS $/ \mathrm{ML}$ robusto } & con item69 & $49(35)$ & 0.977 & 0.977 & 0.031 & 0.036 \\
\hline & con ít & & 0.982 & 0.977 & 025 & 0.036 \\
\hline \multicolumn{2}{|l|}{ AP / WLSMV } & 186 & 0.902 & 0.962 & 24 & 0.054 \\
\hline \multicolumn{2}{|l|}{$\mathrm{AP} / \mathrm{ML}$ robusto } & $131(35)$ & 0.929 & 0.909 & .079 & 0.045 \\
\hline \multicolumn{7}{|c|}{ Todos los items definen un factor general } \\
\hline \multirow[t]{2}{*}{ WLSMV } & con item 69 & $1262(89)$ & 0.563 & 0.779 & 0.173 & 0.14 \\
\hline & & 1257 & 0.558 & 0.774 & 0.169 & 0.137 \\
\hline \multirow[t]{2}{*}{ ML robusto } & con item69 & $1263(89)$ & 0.563 & 0.779 & 0.173 & 0.14 \\
\hline & con & 1753( & 0.592 & 0.562 & 0.097 & 0.107 \\
\hline \multicolumn{7}{|c|}{ Modelo de tres factores } \\
\hline \multirow[t]{2}{*}{ WLSMV } & con item 69 & $319(99)$ & 0.919 & 0.963 & 0.071 & 0.069 \\
\hline & & $348(102)$ & 0.907 & 0.957 & 0.074 & 0.072 \\
\hline \multirow[t]{4}{*}{ ML robusto } & con item 69 & $716(402)$ & 0.907 & 0.9 & 0.042 & 0.054 \\
\hline & con item 3 & 777 (402) & 0.886 & 0.887 & 0.046 & 0.057 \\
\hline & ste aceptable & & $>0.9$ & $>0.9$ & $<0.08$ & $<1$ \\
\hline & Bueno & & $>0.95$ & $>0.95$ & $<0.05$ & $<0.08$ \\
\hline
\end{tabular}

Con respecto a la estructura factorial de la prueba, en la Tabla 3, el escaso ajuste de la solución monofactorial de 30 ítems indica la existencia de más de un factor. Los análisis factor a factor obtienen un buen ajuste (especialmente para el método de estimación WLSMV). No se puede descartar, por tanto, la existencia de esos factores. Pero la cuestión más importante es lo que ocurre cuando factorizamos los 30 ítems conjuntamente y los asignamos a los factores teóricos, el ajuste es también adecuado o bueno.

Tabla 3

Análisis factorial confirmatorio: saturaciones factoriales estandarizadas para el MASQ-30

\begin{tabular}{|c|c|c|c|c|c|c|}
\hline Ítem & \multicolumn{3}{|c|}{ Estimación WLSMV } & \multicolumn{3}{|c|}{ Estimación MLM } \\
\hline & F1 & $\mathrm{F} 2$ & F3 & F1 & F2 & F3 \\
\hline MASQ01 & 0.622 & & & 0.567 & & \\
\hline MASQ04 & 0.693 & & & 0.519 & & \\
\hline MASQ06 & 0.692 & & & 0.635 & & \\
\hline MASQ08 & 0.751 & & & 0.61 & & \\
\hline MASQ10 & 0.699 & & & 0.583 & & \\
\hline MASQ11 & 0.764 & & & 0.617 & & \\
\hline MASQ14 & 0.715 & & & 0.627 & & \\
\hline MASQ21 & 0.674 & & & 0.538 & & \\
\hline MASQ23 & 0.54 & & & 0.524 & & \\
\hline MASQ28 & 0.636 & & & 0.589 & & \\
\hline MASQ02 & & 0.699 & & & 0.5 & \\
\hline MASQ15 & & 0.784 & & & 0.659 & \\
\hline MASQ17 & & 0.549 & & & 0.497 & \\
\hline MASQ18 & & 0.787 & & & 0.627 & \\
\hline MASQ19 & & 0.867 & & & 0.76 & \\
\hline MASQ22 & & 0.871 & & & 0.781 & \\
\hline MASQ25 & & 0.672 & & & 0.59 & \\
\hline MASQ26 & & 0.81 & & & 0.673 & \\
\hline MASQ27 & & 0.773 & & & 0.668 & \\
\hline MASQ30 & & 0.792 & & & 0.631 & \\
\hline MASQ03 & & & 0.595 & & & 0.591 \\
\hline MASQ05 & & & 0.712 & & & 0.678 \\
\hline MASQ07 & & & 0.667 & & & 0.649 \\
\hline MASQ09 & & & 0.696 & & & 0.659 \\
\hline MASQ12 & & & 0.628 & & & 0.615 \\
\hline MASQ13 & & & 0.597 & & & 0.557 \\
\hline MASQ16 & & & 0.409 & & & 0.432 \\
\hline MASQ20 & & & 0.824 & & & 0.738 \\
\hline MASQ24 & & & 0.77 & & & 0.702 \\
\hline MASQ29 & & & 0.774 & & & 0.722 \\
\hline
\end{tabular}

WLSMV - Correlación factores: F1-F2: 0.74;

F1-F3: $-0.51 ;$ F2-F3: -0.34

MLM - Correlación factores: F1-F2: 0.68; F1F3: - $0.48 ;$ F2-F3: -0.3

Fiabilidad: consistencia interna (a de Cronbach) y test-retest

En la Tabla 4, se observa que los coeficientes de consistencia interna para el MASQE30 oscilan entre 0.83 y 0.92 y la fiabilidad test-retest entre 0.41 y 0.62 , siendo en todos los casos muy similares al MASQ90. 
Tabla 4

Consistencia interna ( $\alpha$ de Cronbach) y fiabilidad test-retest $\left(r_{x y}\right)$ para el MASQ-90 y el MASQ-30

\begin{tabular}{lcccccc}
\hline \multirow{2}{*}{ Factor } & \multicolumn{3}{c}{ Consistencia interna $(\alpha)$ de Cronbach } & \multicolumn{2}{c}{ Fiabilidad test-retest } \\
\cline { 2 - 7 } & \multicolumn{2}{c}{$\alpha$} & \multicolumn{2}{c}{$\alpha$} & $r_{\text {xy }}$ & $r_{\mathrm{xy}}$ \\
& \multicolumn{2}{c}{ MASQ90 } & \multicolumn{2}{c}{ MASQE30 } & MASQ90 & MASQE30 \\
\hline \multirow{4}{*}{ Test } & Retest & Test & Retest & \\
AS & $0.96(25)$ & 0.93 & $0.91(10)$ & 0.89 & $0.47^{* * *}$ & $0.41^{* * *}$ \\
AP & $0.93(25)$ & 0.91 & $0.88(10)$ & 0.87 & $0.53^{* * *}$ & $0.49^{* * *}$ \\
AN & $0.97(40)$ & 0.93 & $0.92(10)$ & 0.86 & $0.64^{* * *}$ & $0.62^{* * *}$ \\
MASQ & $0.93(90)$ & 0.91 & $0.83(30)$ & 0.87 & $0.52^{* * *}$ & $0.49^{* * *}$ \\
\hline
\end{tabular}

Nota. AN = Afecto negativo; AS = Ansiedad somática; AP = Afecto positivo; MASQ90

$=$ Puntuación total en el cuestionario; MASQE30 = Puntuación total en el cuestionario; Entre paréntesis el número de ítems. $* * * p \leq 0.001$.

Validez convergente y discriminante: coeficientes de correlación entre los factores del MASQ90 y el MASQE30

En la tabla 5, se pueden observar los coeficientes de correlación interfactores del MASQE30. Así, AS correlaciona $\left(r_{\mathrm{xy}}=-0.24\right)$ con AP y $\left(r_{\mathrm{xy}}=\right.$ $0.6)$ con AN; AP correlaciona $\left(r_{\mathrm{xy}}=-0.38\right)$ con AN. Estos coeficientes son bastantes similares al MASQ90. En la misma tabla se presentan los coeficientes de correlación de los tres factores de las dos escalas.

\section{Tabla 5}

Coeficientes de correlación ( $r$ de Pearson intrafactores de los cuestionarios MASQ90 y MASQE30 y de los primeros con los segundos

\begin{tabular}{lccccc}
\hline \multirow{2}{*}{ Factor } & \multicolumn{2}{c}{ MASQ90 } & \multicolumn{3}{c}{ MASQE30 } \\
\cline { 2 - 6 } & AP & AN & AS & AP & AN \\
\hline AS & $-0.3^{* * *}$ & $0.78^{* * *}$ & & $-0.24^{* * *}$ & $0.6^{* * *}$ \\
AP & & $-0.44^{* * *}$ & & & $-0.38^{* * *}$ \\
AS & & & $0.95^{* * *}$ & $-0.26^{* * *}$ & $0.68^{* * *}$ \\
AP & & & & $0.96^{* * *}$ & $-0.42^{* * *}$ \\
AN & & & & & $0.93^{* * *}$ \\
\hline
\end{tabular}

Nota. AN = Afecto negativo; AS = Ansiedad somática; AP = Afecto positivo; MASQ90

$=$ Puntuación total en el cuestionario; MASQE30 = Puntuación total en el cuestionario. $* * * p \leq 0.00$.

Validez convergente y discriminante entre variables psicopatológicas con las dos versiones del MASQ

En la Tabla 6, se presentan los coeficientes de correlación de orden cero de los tres factores del MASQE30 con variables psicopatológicas. Así, el promedio de coeficientes de correlación de los tres factores con depresión es como sigue: $r_{\mathrm{xy}}=$ 0.46 con AS; $r_{\mathrm{xy}}=-0.41$ con AP y $r_{\mathrm{xy}}=0.61$ con AN. Para la ansiedad, AS correlaciona un promedio de $r_{\mathrm{xy}}=0.63$, con AP es de $r_{\mathrm{xy}}=-0.21$ $\mathrm{y}$ con $\mathrm{AN}$ es de $r_{\mathrm{xy}}=0.58$.

\section{Tabla 6}

Coeficientes de correlación de orden cero entre los factores del MASQ90 y MASQE30 con variables psicopatológicas

\begin{tabular}{|c|c|c|c|c|c|c|}
\hline \multicolumn{7}{|c|}{ Coeficientes de correlación de orden cero } \\
\hline \multirow[t]{2}{*}{ Escala } & \multicolumn{3}{|c|}{ MASQ90 } & \multicolumn{3}{|c|}{ MASQE30 } \\
\hline & AS & AP & AN & AS & AP & AN \\
\hline BDISO & $0.52^{* * *}$ & $-0.35^{* * *}$ & $0.57^{* * *}$ & $0.46^{* * *}$ & $-0.32 * * *$ & $0.51^{* * *}$ \\
\hline BDICA & $0.48^{* * *}$ & -0.46 & & & & \\
\hline BDI-II & $0.55^{* * * *}$ & $-0.45^{* * *}$ & $0.7 * * *$ & $49^{* * * *}$ & $-0.41 * * *$ & 0.66 F \\
\hline BAISU & $0.64 * * *$ & $-0.25 * * *$ & $0.62 * * *$ & $0.59 * * *$ & $-0.22 * * *$ & $0.57 * * *$ \\
\hline & $0.69 * * *$ & & & & & \\
\hline & & & & & & \\
\hline \multicolumn{7}{|c|}{$\begin{array}{l}\text { Nota. } \mathrm{AN}=\text { Afecto negativo; } \mathrm{AS}=\text { Ansiedad } \\
\text { somática; AP = Afecto positivo; BDISO } \\
\text { = Síntomas somáticos de la depresión; } \\
\text { BDICA = Síntomas cognitivo-afectivos de } \\
\text { la depresión; BDI-II = Depresión; BAISUB } \\
\text { = Síntomas cognitivos del pánicos: BAIFI = } \\
\text { síntomas físicos; BAI = Ansiedad subjetiva } \\
\text { y pánico; } * p \leq 0.05 ; * * p \leq 0.001 .\end{array}$} \\
\hline
\end{tabular}

Validez predictiva

Los resultados que se exponen en la Tabla 7 son análisis de regresión múltiple, en los que se toman como predictoras los tres factores del MASQE30 y como variables criterio dos variables de síntomas, a saber: depresión (BDI-II) y ansiedad subjetiva y pánico (BAI). Se empleó la regresión jerárquica. Así, AN es un factor común a los dos trastornos evaluados, con un peso $\beta$ más elevado (0.49) en depresión. La AS es común tanto a la ansiedad como a la depresión, con un peso $\beta$ más alto (0.46) en ansiedad. Finalmente, el AP bajo es especifico de la depresión. 
Tabla 7

Análisis de regresión jerárquica

\begin{tabular}{llccccc}
\hline & & $R^{2}$ & $\Delta R^{2}$ & $\Delta F$ & $\beta$ & $t$ \\
\hline \multirow{2}{*}{ BDI-II } & Paso1: AP & 0.17 & 0.17 & $92.4(1,435)^{* * *}$ & -0.21 & $-5.4^{* * *}$ \\
& Paso2: AN & 0.45 & 0.27 & $217.7(1,434)^{* * *}$ & 0.49 & $10.7^{* * * *}$ \\
& Paso3: AS & 0.46 & 0.01 & $8.3(1,433)^{* * *}$ & 0.13 & $2.87^{* *}$ \\
BAI & Paso1: AS & 0.41 & 0.41 & $290.8(1,412)^{* * *}$ & 0.46 & $10.2^{* * *}$ \\
& Paso2: AN & 0.47 & 0.06 & $48.1(1,411)^{* * * *}$ & 0.31 & $6.5^{* * *}$ \\
\hline
\end{tabular}

Nota. BAI = Agorafobia-pánico; BDI-II =

Depresión; $\mathrm{AN}=$ Afecto Negativo; $\mathrm{AP}=$ Afecto positivo; AS $=$ Ansiedad somática; $\beta=$ el valor

de beta y la probabilidad asociada corresponden

al paso en que se introduce la variable. $* * p \leq 0.01$; $* * * p \leq 0.001 ; * * * p \leq 0.0009$.

\section{Discusión}

Se presenta una versión corta de 30 ítems adaptado del holandés del MASQD30 (Wardenaar et al., 2010) y modificado por los autores de este trabajo del MASQ (Watson et al., 1995), para evaluar su modelo tripartito. Los resultados de los análisis factoriales tanto exploratorio como confirmatorio corroboran una estructura de tres factores cada uno de ellos con 10 ítems, a saber: el afecto negativo (AN), el afecto positivo (AP) y la ansiedad somática (AS), semejantes a la de los autores originales, que confirman la adecuada validez de constructo del MASQE30 en esta muestra de la población general canaria. Por otro lado, parece adecuada la modificación propuesta en el factor de AS, es decir, sustituir el ítem tres "Me sobresalté fácilmente" por el ítem 69 "Mis músculos se crisparon o temblaron". La fiabilidad, entendida como consistencia interna y testretest son altamente satisfactorias y muy similares a los de la muestra holandesa. En lo que se refiere a la validez convergente y discriminante del MASQE30 con trastornos de ansiedad y del estado de ánimo los datos son adecuados (Wardenaar et al., 2010).

Tal y como formula el modelo tripartito, el afecto positivo bajo (AP) es específico de la depresión, que se confirma en este trabajo, pero también se evidencia que AS se relaciona con depresión, dato discordante con el modelo. En este sentido, algunas investigaciones también han encontrado apoyo para una relación significativa entre la AS y la depresión, en contraposición al modelo tripartito
(Chorpita \& Daleiden, 2002). Por otra parte, la AS no distingue entre pacientes con y sin trastorno de ansiedad (Buckby, Yung, Cosgrave, \& Killackey, 2007).

Algunas limitaciones presenta el presente estudio. Una primera es que los 30 ítems de esta versión se extrajeron de los 90 ítems de MASQ. Por otra parte, se realizó una investigación solo con población general, cuando es deseable contar con una amplia muestra de población clínica. En este estudio el porcentaje de mujeres duplica al de hombres, por lo que se proponen estudios posteriores en los que se equipare la muestra por género, así como el análisis de la invariancia factorial por género. Los análisis factoriales exploratorios y confirmatorios se realizan con la misma nuestra, aunque un estudio posterior de los mismos autores confirma una validez de constructo similar a la encontrada aquí.

Las vulnerabilidades temperamentales mencionadas al inicio se sitúan en un orden superior, que aunque necesarias, no son suficientes para explicar la etiología y mantenimiento de los diferentes trastornos de ansiedad y del humor, haciéndose necesario el estudio de vulnerabilidades cognitivas que pudieran ser transdiagnósticas a esos trastornos, y que estarían en un orden inferior, tales como la intolerancia a la incertidumbre, la rumia, la preocupación, la sensibilidad a la ansiedad y el perfeccionismo.

\section{Referencias}

Anderson, E. R., \& Hope, D. A. (2008). A review of the tripartite model for understanding the link between anxiety and depression in youth. Clinical Psychology Review, 28,275-287. http://dx.doi.org/10.1016/ j.cpr.20 07.05.004

Beck, A. T., Epstein, N., Brown, G., \& Steer, R. A. (1988). An inventory for measuring clinical anxiety: Psychometric properties. Journal of Consulting and Clinical Psychology, 56(6), 893-897. http://dx.doi.org/10.1037/ 0022-006X.56.6.893 
Beck, A. T., Steer, R. A., \& Brown, T. A. (1996). Beck Depression Inventory-Manual (2a. ed.). San Antonio: Psychological Corporation.

Boschen, M. J., \& Oei, T. P. (2007). Discriminant validity of the MASQ in a clinical sample. Psychiatry Research, 150, 163-71. http://dx. doi.org/10.1016/j.psychres.2006.03.008

Buckby, J. A., Cotton, S. M., Cosgrave, E. M., Killackey, E. J., \& Yung, A. R. (2008). A factor analytic investigation of the tripartite model of affect in a clinical sample of young Australians. BMC Psychiatry, 8, 79. http://d x.doi.org/10.1186/1471-244X-8-79

Buckby, J.A., Yung, A.R., Cosgrave, E.M., Killackey, E.J. (2007). Clinical utility of the Mood and Anxiety Symptom Questionnaire (MASQ) in a sample of young helpseekers. BMC Psychiatry 7(50). $\mathrm{h}$ ttp://dx.doi.org/10.1186/1471-244X-7-50

Casillas, A., \& Clark, L. A. (mayo, 2000). The Mini Mood and Anxiety Symptom Questionnaire (Mini-MASQ). Poster presentado en la 72 Annual Meeting of the Midwestern Psychological Association, Chicago, IL.

Chorpita, B. F., \& Daleiden, E. L. (2002). Tripartite dimensions of emotion in a child clinical sample: Measurement strategies and implications for clinical utility. Journal of Consulting and Clinical Psychology, 70(5), 1150-1160. http://dx.doi.org/10.1037/0022 $-006 X .70 .5 .1150$

Clark, L. A., \& Watson, D. (1991a). Tripartite model of anxiety and depression: Psychometric evidence and taxonomic implications. Journal of Abnormal Psychology, 100 (3), 316-336. http://dx.doi.o $\mathrm{rg} / 10.1037 / 0021-843 \mathrm{X} .100 .3 .316$

Clark, L. A., \& Watson, D. (1991b). Theoretical and empirical issues in differentiating depression from anxiety. En J. Becker \& A. Kleinman (Eds.), Psychological aspects of mood disorders (pp. 39-65). Hillsdale, NJ: Erlbaum.

de Beurs, E., den Hollander-Gijsman, M. E., Helmich, S., \& Zitman, F. G. (2007). The tripartite model for assessing symptoms of anxiety and depression: Psychometrics of the Dutch version of the mood and anxiety symptoms questionnaire. Behaviour Research and Therapy, 45, 1609-1617. http: //dx.doi.org/10.1016/j.brat.2006.07.004

Egan, S. J., Wade, T. D., \& Shafran, R. (2011). Perfectionism as a transdiagnostic process: A clinical review. Clinical Psychology Review, 31, 203-212. http://dx.doi.org/10.1016/j.cp r.2010.04.009

Ehrenreich-May, J., \& Bilek, E. L. (2012). The development of a transdiagnostic, cognitive behavioral group intervention for childhood anxiety disorders and cooccuring depression symptoms. Cognitive and Behavioral Practice, 19(1), 41-55. http:/ /dx.doi.org/10.1016/j.cbpra.2011.02.003

González, M., Herrero, M., Viña, C. M., Ibáñez, I., \& Peñate, W. (2004). El modelo tripartito: relaciones conceptuales y empíricas entre ansiedad, depresión y afecto negativo. Revista Latinoamericana de Psicología, 36, 289-304. Recuperado de http ://www.redalyc.org/pdf/805/80536208.pdf

Horn, J. L. (1965). A rationale and test for the number of factors in factor analysis. Psychometrica, 30, 179-185. http://dx.doi.or $\mathrm{g} / 10.1007 / \mathrm{BF} 02289447$

Hu, L., \& Bentler, P. M. (1999). Cutoff criteria for fit indexes in covariance structure analysis: Conventional criteria versus new alternatives. Structural Equation Modeling, 6, 1-55. http://dx.doi.org/10.1080/1070551 9909540118

Kessler, R. C., Berglund, P., Demler, O., Jin, R., Merikangas, K. R., \& Walters, E. E. (2005). Lifetime prevalence and age-ofonset distributions of DSM-IV disorders in the National Comorbidity Survey Replication. Archives of General Psychiatry, 62 (6), 593-602. http://dx.doi.org/10.1001/a rchpsyc.62.6.593

Marsh, H., Hau, K., \& Wen, Z. (2004). In search of golden rules: Comment on hypothesis-testing approaches to setting cutoff values for fit indexes and dangers of overgeneralizing $\mathrm{Hu}$ and Bentler's (1999) findings. Structural Equation Modeling, 11, 
320-341. http://dx.doi.org/10.1207/s15328 007sem1103_2

Mineka, S., Watson, D., \& Clark, L. A. (1998). Comorbidity of anxiety and unipolar mood disorders. Anual Review of Psychology, 49, 377-412. http://dx.doi.org/10.1146/annure v.psych.49.1.377

Muthen, L., \& Muthen, B. (1998). Mplus user's guide. Los Angeles, CA: Muthen \& Muthen.

Organización Mundial de la Salud. (2001). Salud mental: nuevos conocimientos, nuevas esperanzas (Informe Mundial de la Salud). Ginebra: Autor.

Reardon, J. M., \& Williams, N. L. (2007). The specificity of cognitive vulnerabilities to emotional disorders: Anxiety sensitivity, looming vulnerability and explanatory style. Journal of Anxiety Disorders, 21, 625-643. http://dx.doi.org/10.1016/j.janxdi s.2006.09.013

Sanz, J., Navarro, M. E., \& Vázquez, C. (2003). Adaptación española del Inventario para la Depresión de Beck-II (BDI-II): 1. Propiedades psicométricas en estudiantes universitarios. Análisis y Modificación de Conducta, 29, 239-288. Recuperado de https://dialnet.unirioja.es/servlet/articul $\mathrm{o}$ ? codigo $=761288$

Satorra, A., \& Bentler, P. M. (1994). Corrections to test statistics and standard errors in covariance structure analysis. En A. von Eye \& C. C. Clogg (Eds.), Latent variables analysis: Applications to developmental research (pp. 399-419). Thousand Oaks, CA: Sage.

Tellegen, A. (1985). Structures of mood and personality and their relevance to assessing anxiety, with emphasis on self-report. En A. H. Tuma \& J. D. Maser (Eds.), Anxiety and the anxiety disorders (pp. 681-706). Hillsdale, NJ: LEA.

Velicer, W. F. (1976). Determining the number of components from the matrix of partial correlations. Psychometrika, 41, 321-327. ht tp://dx.doi.org/10.1007/BF02293557

Velicer, W. F., Eaton, C. A., \& Fava, J. L. (2000). Construct explication through factor or component analysis: A review and evaluation of alternative procedures for determining the number of factors or components. En R. D. Goffin \& E. Helmes (Eds.), Problems and solutions in human assessment: Honoring Douglas N. Jackson at seventy (pp. 41-71). Norwell, MA: Kluwer Academic. http://dx.doi.org/10.1007/978-1 $-4615-4397-8$ 3

Wardenaar, K. J., van Veen, T., Giltay, E. J., de Beurs, E., Penninx, W. J. H., \& Zitman, F. G. (2010). Development and validation of a 30-item short adaptation of the Mood and Anxiety Symptoms Questionnaire (MASQ). Psychiatry Research, 179, 101-106. http://dx.doi.org/10.1016/j.psychr es.2009.03.005

Watson, D., Clark, L. A., Weber, K., Assenheimer, J. S., Strauss, M. E., \& McCormick, R. A. (1995). Testing a tripartite model: II. Exploring the symptom structure of anxiety and depression in student, adult, and patient samples. Journal of Abnormal Psychology, 104(1), 15-25. http ://dx.doi.org/10.1037/0021-843X.104.1.15

\section{Notas}

* Artículo de investigación. Subvencionado por el Vicerrectorado de Investigación de la Universidad de La Laguna (ULL). 